\title{
THE LAMBDA-PROPERTY FOR GENERALISED DIRECT SUMS OF NORMED SPACES
}

\author{
Robert H. Lohman and Thaddeus J. Shura
}

\begin{abstract}
This paper considers direct sums of normed spaces with respect to a Banach space with a normalised, unconditionally strictly monotone basis. Necessary and sufficient conditions are given for such direct sums to have the $\lambda$-property. These results are used to construct examples of reflexive Banach spaces $U$ and $V$ such that $U$ has the uniform $\lambda$-property but $U^{*}$ fails to have the $\lambda$-property, while $V$ and $V^{*}$ fail to have the $\lambda$-property.
\end{abstract}

If $X$ is a normed space and $x$ is in the closed unit ball $B_{X}$ of $X$, a triple $(e, y, \lambda)$ is said to be amenable to $x$ in case $e \in$ ext $\left(B_{X}\right), y \in B_{X}, 0<\lambda \leqslant 1$ and $x=$ $\lambda e+(1-\lambda) y$. In this case, the number $\lambda(x)$ is defined by

$$
\lambda(x)=\sup \{\lambda:(e, y, \lambda) \text { is amenable to } x\} .
$$

$X$ is said to have the $\lambda$-property if each $x \in B_{X}$ admits an amenable triple. If $X$ has the $\lambda$-property and $\lambda(X) \equiv \inf \left\{\lambda(x): x \in B_{X}\right\}>0$, then $X$ is said to have the uniform $\lambda$-property .

General facts and geometric ramifications concerning the $\lambda$-property can be found in [1] and [4]. It is now known that many different types of classical sequence and function spaces have the $\lambda$-property or uniform $\lambda$-property (see $[1-3,5,7,10])$. In this paper, our goal is to consider generalised direct sums of the form $\left(\oplus \sum_{k=1}^{\infty} X_{k}\right)$, where $Z$ is a Banach space with a normalised, unconditionally strictly monotone basis and $\left(X_{k}\right)$ is a sequence of normed spaces. We give necessary and sufficient conditions for such spaces to have the $\lambda$-property (Theorem 8, Corollary 10) or to have the uniform $\lambda$-property (Theorem 9, Corollary 11). In particular, our results generalise Theorem 3 of [5], which considered $\left(\oplus \sum_{k=1}^{\infty} X_{k}\right)_{\ell_{1}}$. Using our results, we are able to give examples of reflexive Banach spaces $U$ and $V$ such that $U$ has the uniform $\lambda$-property but $U^{*}$ fails to have the $\lambda$-property, while $V$ and $V^{*}$ both fail to have the $\lambda$-property .

Received 29th June, 1989.

Copyright Clearance Centre, Inc. Serial-fee code: 0004-9729/90 \$A2.00+0.00. 


\section{Notation}

Throughout the paper, $Z$ denotes a Banach space with a normalised unconditional basis $\left(z_{k}\right)$. The basis $\left(z_{k}\right)$ is said to be unconditionally monotone in case $\sum_{k=1}^{\infty} a_{k} z_{k}, \sum_{k=1}^{\infty} b_{k} z_{k} \in Z$ and $\left|a_{k}\right| \leqslant\left|b_{k}\right|$ for all $k$ imply

$$
\left\|\sum_{k=1}^{\infty} a_{k} z_{k}\right\| \leqslant\left\|\sum_{k=1}^{\infty} b_{k} z_{k}\right\|
$$

If, in addition, $\left|a_{k}\right|<\left|b_{k}\right|$ for some $k$ implies strict inequality in (1), then $\left(z_{k}\right)$ is said to be unconditionally strictly monotone. For example, the standard unit vector bases of $\ell_{p}, 1 \leqslant p<\infty$, are unconditionally strictly monotone, while the standard unit vector basis of $c_{0}$ is not. Throughout the paper, it is assumed that $\left(z_{k}\right)$ is unconditionally strictly monotone. $S_{X}$ denotes the unit sphere of a normed space $X$. If $X$ and $Y$ are normed spaces, we write $X \cong Y$ if $X$ is isometrically isomorphic to $Y$. space

Given a sequence $\left(X_{k}\right)$ of normed spaces, $X=\left(\oplus \sum_{k=1}^{\infty} X_{k}\right)_{Z}$ denotes the normed

$$
\left\{x=\left(x_{k}\right): x_{k} \in X_{k} \text { for all } k \text { and } \sum_{k=1}^{\infty}\left\|x_{k}\right\| z_{k} \in Z\right\}
$$

with $\|x\|$ defined by

$$
\|x\|=\left\|\sum_{k=1}^{\infty}\right\| x_{k}\left\|z_{k}\right\|
$$

If each space $X_{k}$ has the $\lambda$-property, we denote its $\lambda$-function by $\lambda_{k}$. In this case, if $x=\left(x_{k}\right) \in B_{X}$ and $x \neq 0$, we write

$$
\Lambda(x)=\inf \left\{\lambda_{k}\left(\frac{x_{k}}{\left\|x_{k}\right\|}\right): x_{k} \neq 0\right\}
$$

1. The $\lambda$-Property IN $\left(\oplus \sum_{k=1}^{\infty} X_{k}\right)_{Z}$

In order to investigate the $\lambda$-property for the normed space $X=\left(\oplus \sum_{k=1}^{\infty} X_{k}\right)_{Z}$, it is necessary to have a description of the extreme points of $B_{X}$. We wish to thank Professor Pei-Kee Lin for pointing out such a description (Lemma 1) and for suggesting investigation of the $\lambda$-property in general spaces $X$ as above. The proof of the following lemma is essentially the same as that for the case $Z=\ell_{p}$ (see [9]) and is omitted. 
LEMMA 1. If $x=\left(x_{k}\right) \in B_{X}$, the following are equivalent:

(a) $x \in \operatorname{ext}\left(B_{X}\right)$

(b) $\sum_{k=1}^{\infty}\left\|x_{k}\right\| z_{k} \in \operatorname{ext}\left(B_{Z}\right)$ and $x_{k} /\left\|x_{k}\right\| \in \operatorname{ext}\left(B_{X_{k}}\right)$, if $x_{k} \neq 0$.

REMARK 2. Let $\left(\varepsilon_{k}\right)$ be a sequence of scalars with $\left|\varepsilon_{k}\right|=1$ for all $k$.

The mapping $T: Z \rightarrow Z$, defined by

$$
T\left(\sum_{k=1}^{\infty} a_{k} z_{k}\right)=\sum_{k=1}^{\infty} \varepsilon_{k} a_{k} z_{k},
$$

is a linear isometry of $Z$ onto $Z$. In particular, $z \in \operatorname{ext}\left(B_{Z}\right)$ if and only if $T(z) \in$ ext $\left(B_{Z}\right)$.

LEMMA 3. Assume that each summand $X_{k}$ has the $\lambda$-property and $x=\left(x_{k}\right) \epsilon$ $S_{X}$. If $\sum_{k=1}^{\infty}\left\|x_{k}\right\| z_{k}$ admits an amenable triple $\left(\sum_{k=1}^{\infty} a_{k} z_{k}, \sum_{k=1}^{\infty} b_{k} z_{k}, \lambda\right)$ and $\Lambda(x)>0$, then $x$ admits an amenable triple and $\lambda(x) \geqslant \lambda \Lambda(x)$.

Proof: Let $0<\alpha<\Lambda(x)$. If $x_{k} \neq 0$, then $\alpha<\Lambda(x) \leqslant \lambda_{k}\left(x_{k} /\left\|x_{k}\right\|\right)$. By Proposition 1.2 of $[1]$, there is a triple $\left(e_{k}, y_{k}^{\prime}, \alpha\right)$ amenable to $x_{k} /\left\|x_{k}\right\|$. If $x_{k}=0$, define $e_{k}=y_{k}^{\prime}=0$. Then for all $k$, we have

$$
x_{k}=\alpha\left\|x_{k}\right\| e_{k}+(1-\alpha)\left\|x_{k}\right\| y_{k}^{\prime} .
$$

Since $\sum_{k=1}^{\infty}\left\|x_{k}\right\| z_{k}=\sum_{k=1}^{\infty}\left[\lambda a_{k}+(1-\lambda) b_{k}\right] z_{k}$, we obtain

$$
\left\|x_{k}\right\|=\lambda a_{k}+(1-\lambda) b_{k}
$$

for all $k$. By (2) and (3), write $x_{k}=(\alpha \lambda) a_{k} e_{k}+(1-\alpha \lambda) y_{k}$, where

$$
y_{k}=\frac{\alpha(1-\lambda) b_{k} e_{k}+(1-\alpha) \lambda a_{k} y_{k}^{\prime}+(1-\alpha)(1-\lambda) b_{k} y_{k}^{\prime}}{1-\alpha \lambda} .
$$

Next, observe that all of the sequences $\left(a_{k} e_{k}\right),\left(b_{k} e_{k}\right),\left(a_{k} y_{k}^{\prime}\right),\left(b_{k} y_{k}^{\prime}\right)$ are in $B_{X}$ (for example, $\left.\left\|\left(a_{k} e_{k}\right)\right\|=\left\|\sum_{k=1}^{\infty}\right\| a_{k} e_{k}\left\|z_{k}\right\| \leqslant\left\|\sum_{k=1}^{\infty} a_{k} z_{k}\right\|=1\right)$. Thus, if $y=\left(y_{k}\right)$, we have

$$
\|y\| \leqslant \frac{\alpha(1-\lambda)\left\|\left(b_{k} e_{k}\right)\right\|+(1-\alpha) \lambda\left\|\left(a_{k} y_{k}^{\prime}\right)\right\|+(1-\alpha)(1-\lambda)\left\|\left(b_{k} y_{k}^{\prime}\right)\right\|}{1-\alpha \lambda} \leqslant 1 .
$$

Letting $e=\left(a_{k} e_{k}\right)$, we see that $\|e\| \leqslant 1$. Since $x=\alpha \lambda e+(1-\alpha \lambda) y, 0<\alpha \lambda<1$ and $\|x\|=1$, we must have $\|e\|=\|y\|=1$. Therefore,

$$
1=\|e\|=\left\|\sum_{k=1}^{\infty}\right\| a_{k} e_{k}\left\|z_{k}\right\| \leqslant\left\|\sum_{k=1}^{\infty}\left|a_{k}\right| z_{k}\right\|=1
$$


By strict monotonicity, $\left\|a_{k} e_{k}\right\|=\left|a_{k}\right|$ for all $k$. Consequently, $\sum_{k=1}^{\infty}\left\|a_{k} e_{k}\right\| z_{k}=$ $\sum_{k=1}^{\infty}\left|a_{k}\right| z_{k}$. By hypothesis $\sum_{k=1}^{\infty} a_{k} z_{k} \in \operatorname{ext}\left(B_{Z}\right)$. Remark 2 yields $\sum_{k=1}^{\infty}\left|a_{k}\right| z_{k} \in \operatorname{ext}\left(B_{Z}\right)$. Also, if $a_{k} e_{k} \neq 0$, then $e_{k} \in \operatorname{ext}\left(B_{X_{k}}\right)$ and $a_{k} e_{k} /\left\|a_{k} e_{k}\right\|=\varepsilon_{k} e_{k}$, where $\left|\varepsilon_{k}\right|=1$. Therefore, $a_{k} e_{k} /\left\|a_{k} e_{k}\right\| \in \operatorname{ext}\left(B_{X_{k}}\right)$ whenever $a_{k} e_{k} \neq 0$. By Lemma 1, $e \in \operatorname{ext}\left(B_{X}\right)$. This shows $(e, y, \alpha \lambda)$ is amenable to $x$ and establishes the fact that $\lambda(x) \geqslant \alpha \lambda$. Taking the supremum over all such $\alpha$ establishes $\lambda(x) \geqslant \lambda \Lambda(x)$.

TheOREM 4. Assume that $X_{k}, k=1,2, \ldots$, and $Z$ have the $\lambda$-property . If there exists a subset $N_{0}$ of $N$, with finite complement, such that $\inf _{k \in N_{0}} \lambda_{k}\left(X_{k}\right)>0$, then

(i) $X=\left(\oplus \sum_{k=1}^{\infty} X_{k}\right)_{Z}$ has the $\lambda$-property .

(ii) If $0 \neq x=\left(x_{k}\right) \in B_{X}$,

$$
\lambda(x) \geqslant \frac{1+\|x\|}{2} \Lambda(x) \lambda\left(\sum_{k=1}^{\infty} \frac{\left\|x_{k}\right\|}{\|x\|} z_{k}\right) .
$$

Proof: Let $0 \neq x=\left(x_{k}\right) \in B_{X}$. Then $x /\|x\|=\left(x_{k}\right) /\|x\| \in S_{X}$ and $\sum_{k=1}^{\infty}\left(\left\|x_{k}\right\| /\|x\| z_{k}\right)$ admits an amenable triple $\left(\sum_{k=1}^{\infty} a_{k} z_{k}, \sum_{k=1}^{\infty} b_{k} z_{k}, \lambda\right)$, where $\lambda \leqslant \lambda\left(\sum_{k=1}^{\infty}\left(\left\|x_{k}\right\| /\|x\|\right) z_{k}\right)$. Since $\inf _{k \in N_{0}} \lambda_{k}\left(X_{k}\right)>0$, it follows that $\Lambda(x)>0$. By Lemma $3, x /\|x\|$ admits an amenable triple and

$$
\lambda(x /\|x\|) \geqslant \lambda \Lambda(x /\|x\|)=\lambda \Lambda(x) .
$$

Taking the supremum over all such $\lambda$ shows

$$
\lambda\left(\frac{x}{\|x\|}\right) \geqslant \Lambda(x) \lambda\left(\sum_{k=1}^{\infty} \frac{\left\|x_{k}\right\|}{\|x\|} z_{k}\right) .
$$

By the proof of Lemma 2.1 of [1], $x$ admits an amenable triple, establishing (i), and $\lambda(x) \geqslant((1+\|x\|) / 2) \lambda(x /\|x\|)$, establishing (ii).

In order to obtain a converse of Theorem 4 , we need $\operatorname{ext}\left(B_{Z}\right)$ to have a diversity of extreme points.

DEFINITION 5. The extreme points of $B_{Z}$ are said to be diversified if for each increasing sequence $\left(k_{n}\right)$ in $N, B_{Z}$ has an extreme point of the form $\sum_{n=1}^{\infty} a_{n} z_{k_{n}}$, where $a_{n} \neq 0$ for all $n$. 
REMARK 6. There are many different conditions under which the extreme points of $B_{Z}$ are diversified. Let $\left(k_{n}\right)$ be an increasing sequence in N. If $Z$ is strictly convex, let $w=\sum_{n=1}^{\infty}\left(z_{k_{n}}\right) /\left(2^{n}\right)$. Then $w /\|w\|$ is an extreme point of $B_{Z}$ of the form required in Definition 5. Also, if $Z$ is a symmetric space (see [8]) and $B_{Z}$ contains an extreme point $\sum_{k=1}^{\infty} a_{k} z_{k}$ with infinite support, let the nonzero $a_{k}$ 's be indexed by $j_{1}<j_{2}<\cdots$. Then the vector $\sum_{n=1}^{\infty} a_{j_{n}} z_{k_{n}}$ is an extreme point of $B_{Z}$. Finally, if the extreme points of $B_{Z}$ are diversified, then for each increasing sequence $\left(k_{n}\right)$ in $N$, Remark 2 guarantees that there exists $\sum_{n=1}^{\infty} a_{n} z_{k_{n}} \in \operatorname{ext}\left(B_{Z}\right)$ with $a_{n}>0$ for all $n$.

Theorem 7. Assume $X=\left(\oplus \sum_{k=1}^{\infty} X_{k}\right)_{Z}$ has the $\lambda$-property . Then:

(i) Each summand $X_{k}$ has the $\lambda$-property .

(ii) $Z$ has the $\lambda$-property .

(iii) If, in addition, the extreme points of $B_{Z}$ are diversified, there exists a subset $N_{0}$ of $N$, with finite complement, such that $\inf _{k \in N_{0}} \lambda_{k}\left(X_{k}\right)>0$.

Proof: (i) We show that $X_{1}$ has the $\lambda$-property (the proof for other indices is the same). If $x_{1} \in S_{X_{1}}$, define $x=\left(x_{1}, 0,0, \ldots\right)$. By hypothesis, we can write $x=\lambda e+(1-\lambda) y$, where $e=\left(e_{k}\right) \in \operatorname{ext}\left(B_{X}\right), y=\left(y_{k}\right) \in B_{X}, 0<\lambda \leqslant 1$. If $\lambda=1$, then $x=e$ and $x_{1}=e_{1} \in \operatorname{ext}\left(B_{X_{1}}\right)$ by Lemma 1. If $0<\lambda<1$, then $x_{1}=\lambda e_{1}+(1-\lambda) y_{1}$ forces $\left\|e_{1}\right\|=\left\|y_{1}\right\|=1$. By strict monotonicity, $e_{k}=y_{k}=0$ for $k \geqslant 2$. Then $e_{1} \in \operatorname{ext}\left(B_{X_{1}}\right)$ and $\left(e_{1}, y_{1}, \lambda\right)$ is amenable to $x_{1}$. Since unit vectors in $X_{1}$ admit amenable triples, the proof of Lemma 2.1 of [1] shows that $X_{1}$ has the $\lambda$-property .

(ii) It suffices to show that each $z=\sum_{k=1}^{\infty} a_{k} z_{k} \in S_{Z}$ admits an amenable triple. By Remark 2, we may assume $a_{k} \geqslant 0$ for all $k$. For each $k$, choose $e_{k} \in \operatorname{ext}\left(B_{X_{k}}\right)$ and define $x=\left(a_{k} e_{k}\right)$. Then $\|x\|=\|z\|=1$. We can write $x=\lambda e+(1-\lambda) y$, where $e=\left(v_{k}\right) \in \operatorname{ext}\left(B_{X}\right), y=\left(y_{k}\right) \in S_{X}$ and $0<\lambda \leqslant 1$. If $\lambda=1$, then $x=e$ and Lemma 1 yields $z \in$ ext $\left(B_{Z}\right)$. Thus, we may assume $0<\lambda<1$. For all $k$, we have

$$
a_{k} e_{k}=\lambda v_{k}+(1-\lambda) y_{k}
$$


Therefore,

$$
\begin{aligned}
1=\|x\|=\left\|\sum_{k=1}^{\infty} a_{k} z_{k}\right\| & =\left\|\sum_{k=1}^{\infty}\right\| \lambda v_{k}+(1-\lambda) y_{k}\left\|z_{k}\right\| \\
& \leqslant\left\|\sum_{k=1}^{\infty}\left[\lambda\left\|v_{k}\right\|+(1-\lambda)\left\|y_{k}\right\|\right] z_{k}\right\| \\
& \leqslant \lambda\left\|\sum_{k=1}^{\infty}\right\| v_{k}\left\|z_{k}\right\|+(1-\lambda)\left\|\sum_{k=1}^{\infty}\right\| y_{k}\left\|z_{k}\right\| \\
& =1 .
\end{aligned}
$$

By strict monotonicity,

$$
a_{k}=\left\|a_{k} e_{k}\right\|=\lambda\left\|v_{k}\right\|+(1-\lambda)\left\|y_{k}\right\|
$$

for all $k$. Consequently,

$$
z=\lambda\left(\sum_{k=1}^{\infty}\left\|v_{k}\right\| z_{k}\right)+(1-\lambda)\left(\sum_{k=1}^{\infty}\left\|y_{k}\right\| z_{k}\right) .
$$

Since $\sum_{k=1}^{\infty}\left\|v_{k}\right\| z_{k} \in \operatorname{ext}\left(B_{Z}\right)$ by Lemma 1, it follows that $\left(\sum_{k=1}^{\infty}\left\|v_{k}\right\| z_{k}, \sum_{k=1}^{\infty}\left\|y_{k}\right\| z_{k}, \lambda\right)$ is amenable to $z$.

(iii) Assume, to the contrary, that no such set $\mathrm{N}_{0}$ exists. Then there exist $k_{1}<$ $k_{2}<\cdots$ with $\lambda_{k_{n}}\left(X_{k_{n}}\right) \rightarrow 0$. Therefore, we can choose $u_{k_{n}} \in S_{X_{k_{n}}}$ such that $\lambda_{k_{n}}\left(u_{k_{n}}\right) \rightarrow 0$. By hypothesis, there exists $\sum_{n=1}^{\infty} a_{k_{n}} z_{k_{n}} \in \operatorname{ext}\left(B_{Z}\right)$ with $a_{k_{n}}>0$ for all $n$. If $k \notin\left\{k_{1}, k_{2}, \ldots\right\}$, define $a_{k}=0, u_{k}=0$. Then $x \equiv\left(a_{k} u_{k}\right) \in S_{X}$.

We can write $x=\lambda e+(1-\lambda) y$, where $0<\lambda \leqslant 1, e=\left(v_{k}\right) \in \operatorname{ext}\left(B_{X}\right), y=$ $\left(y_{k}\right) \in S_{X}$. If $\lambda=1$, then $x=e$ and, by Lemma $1, u_{k_{n}} \in \operatorname{ext}\left(B_{X_{k_{n}}}\right)$ for all $n$, which contradicts $\lambda_{k_{n}}\left(u_{k_{n}}\right) \rightarrow 0$. Thus, $0<\lambda<1$ and, as in the proof of (ii), we obtain

$$
a_{k}=\lambda\left\|v_{k}\right\|+(1-\lambda)\left\|y_{k}\right\|
$$

for all $k$. In particular, $v_{k}=y_{k}=0$ for $k \notin\left\{k_{1}, k_{2}, \ldots\right\}$. Therefore,

$$
\sum_{n=1}^{\infty} a_{k_{n}} z_{k_{n}}=\lambda\left(\sum_{n=1}^{\infty}\left\|v_{k_{n}}\right\| z_{k_{n}}\right)+(1-\lambda)\left(\sum_{n=1}^{\infty}\left\|y_{k_{n}}\right\| z_{k_{n}}\right) .
$$


Since $\sum_{n=1}^{\infty} a_{k_{n}} z_{k_{n}} \in \operatorname{ext}\left(B_{Z}\right)$, we must have

$$
\begin{aligned}
\sum_{n=1}^{\infty} a_{k_{n}} z_{k_{n}} & =\sum_{n=1}^{\infty}\left\|v_{k_{n}}\right\| z_{k_{n}}=\sum_{n=1}^{\infty}\left\|y_{k_{n}}\right\| z_{k_{n}}, \\
a_{k_{n}} & =\left\|v_{k_{n}}\right\|=\left\|y_{k_{n}}\right\|
\end{aligned}
$$

or

for all $n$. Therefore, for all $n$

$$
u_{k_{n}}=\lambda \frac{v_{k_{n}}}{\left\|v_{k_{n}}\right\|}+(1-\lambda) \frac{y_{k_{n}}}{\left\|y_{k_{n}}\right\|}
$$

But $v_{k_{n}} /\left\|v_{k_{n}}\right\| \in \operatorname{ext}\left(B_{X_{k_{n}}}\right)$ implies $\lambda_{k_{n}}\left(u_{k_{n}}\right) \geqslant \lambda$ for all $n$, a contradiction.

Combining Theorems 4 and 7, we obtain

ThEOREM 8. Assume that the extreme points of $B_{Z}$ are diversified. The following are equivalent:

(a) $X=\left(\oplus \sum_{k=1}^{\infty} X_{k}\right)_{Z}$ has the $\lambda$-property .

(b) Each space $X_{k}$ has the $\lambda$-property, there exists a subset $\mathrm{N}_{0}$ of $\mathrm{N}$, with finite complement, such that $\inf _{k \in N_{0}} \lambda_{k}\left(X_{k}\right)>0$, and $Z$ has the $\lambda$-property

We now turn our attention to the uniform $\lambda$-property

THEOREM 9. The following are equivalent:

(a) $X=\left(\oplus \sum_{k=1}^{\infty} X_{k}\right)_{Z}$ has the uniform $\lambda$-property .

(b) Each summand $X_{k}$ has the uniform $\lambda$-property, $\Lambda \equiv \underset{k}{\inf } \lambda_{k}\left(X_{k}\right)>0$ and $Z$ has the uniform $\lambda$-property .

In this case, we have

$$
\lambda(X) \geqslant \frac{\Lambda}{2} \lambda(Z)
$$

Proof: $(a) \Rightarrow(b)$. By Theorem 7 , each summand $X_{k}$ has the $\lambda$-property . Moreover, the proof of Theorem 7 shows that if $x_{k} \in S_{X_{k}}$, then

$$
\lambda_{k}\left(x_{k}\right) \geqslant \lambda\left(0, \ldots, 0, x_{k}, 0, \ldots\right) \geqslant \lambda(X)>0
$$

It follows from Lemma 2.1 of [1] that $\Lambda>0$.

(b) $\Rightarrow(a)$. This follows from Theorem 4 , as does the asserted inequality.

In case the summands are the same, we can sharpen our results as follows. 
Corollary 10. Let $X_{k}=Y$ for all $k$ and assume $B_{Z}$ contains an extreme point with infinite support. The following are equivalent:

(a) $X=\left(\oplus \sum_{k=1}^{\infty} Y\right)_{Z}$ has the $\lambda$-property .

(b) $Y$ has the uniform $\lambda$-property and $Z$ has the $\lambda$-property.

Proof: $(b) \Rightarrow(a)$. This follows from Theorem 7 .

(a) $\Rightarrow(b)$. By Theorem 7, $Y$ and $Z$ have the $\lambda$-property. If $Y$ fails to have the uniform $\lambda$-property, there is a sequence $\left(w_{n}\right)$ in $S_{Y}$ with $\lambda\left(w_{n}\right) \rightarrow 0$. By hypothesis, there exists $\sum_{n=1}^{\infty} a_{k_{n}} z_{k_{n}} \in \operatorname{ext}\left(B_{Z}\right)$ with $a_{k_{n}}>0$ for all $n$. Define $a_{k}=0$ if $k \notin$ $\left\{k_{1}, k_{2}, \ldots\right\}$ and let

$$
u_{k}= \begin{cases}w_{n}, & \text { if } k=k_{n} \text { for some } n \\ 0, & k \notin\left\{k_{1}, k_{2}, \ldots\right\} .\end{cases}
$$

We can then write $x=\left(a_{k} u_{k}\right)$ and proceed as in the proof of part (iii) of Theorem 7 to obtain the same contradiction as before.

An immediate consequence of Theorem 9 is

Corollary 11. Let $X_{k}=Y$ for all $k$. The following are equivalent:

(a) $X=\left(\oplus \sum_{k=1}^{\infty} Y\right)_{Z}$ has the uniform $\lambda$-property .

(b) $Y$ and $Z$ have the uniform $\lambda$-property .

In this case, $\lambda(X) \geqslant \lambda(Y) \lambda(Z) / 2$.

Combining Corollaries 10 and 11 , we obtain

Corollary 12. Assume that $Y$ has the uniform $\lambda$-property and that $Z$ has the $\lambda$-property but not the uniform $\lambda$-property. If $B_{Z}$ contains an extreme point with infinite support, then $\left(\oplus \sum_{k=1}^{\infty} Y\right)_{Z}$ has the $\lambda$-property but not the uniform $\lambda$-property

The preceding corollary provides us with the following curiosity which one should compare to the well-known fact that $\left(\oplus \sum_{k=1}^{\infty} \ell_{p}\right)_{\ell_{p}} \cong \ell_{p}, 1 \leqslant p<\infty$.

Corollary 13. If $Z$ has the $\lambda$-property but not the uniform $\lambda$-property and $\left(\oplus \sum_{k=1}^{\infty} Z\right)_{Z} \cong Z$, then all the extreme points of $B_{Z}$ have finite support.

REMARK 14. In view of Corollary 12 , it should be noted that there are spaces $Z$ which have the $\lambda$-property, fail to have the uniform $\lambda$-property and for which $B_{Z}$ contains extreme points with infinite support. For example, consider $\ell_{1}$ and $\ell_{2}$ over the reals and let $Z=\left(\ell_{1} \oplus \ell_{2}\right)_{\ell_{2}}$ (that is, $\left.Z \cong\left(\ell_{1} \oplus \mathbf{R} \oplus \mathrm{R} \oplus \cdots\right)_{\ell_{2}}\right)$. Then $Z$ has a normalised 
unconditionally strictly monotone basis and, by Lemma $1, B_{Z}$ contains extreme points with infinite support. On the other hand, $\ell_{1}$ has the $\lambda$-property but not the uniform $\lambda$-property ([1]). Consequently, Theorems 8 and 9 imply that $Z$ has the $\lambda \lambda$-property but not the uniform $\lambda$-property .

\section{REFLEXIVITY AND THE $\lambda$-PROPERTY}

We close with two examples concerning reflexive Banach spaces. It has been conjectured that reflexivity might play a special role in the study of the $\lambda$-property and the uniform $\lambda$-property. We now show that the most natural questions one might pose regarding reflexive spaces and the $\lambda$-property have a negative answer. Consequently, reflexivity does not appear to play any significant role in the study of these properties.

It follows from the results of $[1]$ that $\lambda\left(\ell_{\infty}^{k}\right)=1 / 2$ and $\lambda\left(\ell_{1}^{k}\right) \leqslant 1 / k$ for all $k$. By Theorem 9, the reflexive Banach space

$$
U=\left(\oplus \sum_{k=1}^{\infty} \ell_{\infty}^{k}\right)_{\ell_{2}}
$$

has the uniform $\lambda$-property. Since $U^{*} \cong\left(\oplus \sum_{k=1}^{\infty} \ell_{1}^{k}\right)_{\ell_{2}}$, Theorem 8 shows that $U^{*}$ fails to have the $\lambda$-property (this fact was also obtained in [6] by means of direct calculations rather than a general theorem).

Now let $V=\left(U \oplus U^{*}\right)_{\ell_{2}}$; that is,

$$
V \cong\left(\ell_{\infty}^{1} \oplus \ell_{1}^{1} \oplus \ell_{\infty}^{2} \oplus \ell_{1}^{2} \oplus \cdots\right)_{\ell_{2}} .
$$

Then $V$ is a reflexive Banach space which fails to have the $\lambda$-property by Theorem 8. Since $V^{*} \cong V, V^{*}$ also fails to have the $\lambda$-property. This is the first example of a reflexive Banach space with this property (a nonreflexive Banach space with this property was given in [1]).

Finally, it should be noted that a reflexive Banach space $W$ with the $\lambda$-property does not necessarily have the uniform $\lambda$-property. Such an example is given in [6]. In fact, $B_{W}$ can be constructed from $B_{\ell_{2}}$ with very slight modifications.

\section{REFERENCES}

[1] R.M. Aron and R.H. Lohman, 'A geometric function determined by extreme points of the unit ball of a normed space', Pacific J. Math. 127 (1987), 209-231.

[2] A.S. Granero, 'On the Aron-Lohman $\lambda$-property', (preprint). 
[3] A.S. Granero, 'The $\lambda$-function in the spaces $\left(\oplus \sum_{i \in I} X_{i}\right)_{p}$ and $L_{p}(\mu, X), 1 \leqslant p \leqslant \infty$ ', (preprint).

[4] R.H. Lohman, 'The $\lambda$-function in Banach spaces', Contemporary Mathematics, Banach Space Theory 85 (1989), 345-354.

[5] R.H. Lohman and T.J. Shura, 'Calculation of the $\lambda$-function for several classes of normed linear spaces, Nonlinear and Convex Analysis', in Proceedings in Honor of Ky Fan, Marcel Dekker Lecture Notes in Pure and Applied Mathematics, pp. 167-174, 1987.

[6] T.J. Shura, Ph.D. Dissertation, Kent State University.

[7] T.J. Shura and D. Trautman, 'The $\lambda$-property in Schreier's space $S$ and the Lorentz space $d(a, 1)^{\prime}$, (preprint).

[8] I. Singer, Bases in Banach Spaces I (Springer-Verlag, Heidelberg, Berlin, New York, 1970).

[9] M.A. Smith, 'Rotundity and extremicity in $\ell^{p}\left(X_{i}\right)$ and $L^{p}(\mu, X)$ ', in Contemporary Mathematics: Geometry of Normed Linear Spaces 52, pp. 143-160, 1986.

[10] F.B. Trujillo, 'The $\lambda$-property in the $C(K, \mathbf{R})$ spaces', (preprint).

Department of Mathematical Sciences

Kent State University

Kent, OH 44242

United States of America
Department of Mathematical Sciences

Kent State University at Salem

Salem $O H 44460$

United States of America 